\title{
Entanglement Is Not Necessary for Perfect Discrimination between Unitary Operations
}

\author{
Runyao Duan $*$ Yuan Feng $]^{\dagger}$ and Mingsheng Yingt \\ State Key Laboratory of Intelligent Technology and Systems, \\ Department of Computer Science and Technology, Tsinghua University, Beijing, China, 100084
}

(Dated: February 16, 2018)

\begin{abstract}
We show that a unitary operation (quantum circuit) secretely chosen from a finite set of unitary operations can be determined with certainty by sequentially applying only a finite amount of runs of the unknown circuit. No entanglement or joint quantum operations is required in our scheme. We further show that our scheme is optimal in the sense that the number of the runs is minimal when discriminating only two unitary operations.

PACS numbers: $\quad$ 03.65.Ta, 03.65.Ud, 03.67.-a
\end{abstract}

Entanglement is a valuable physical resource for accomplishing many useful quantum computing and quantum information processing tasks [1]. For certain tasks such as superdense coding [2] and quantum teleportation [3], it has been demonstrated that entanglement is an indispensable ingredient. For many other tasks entanglement is also used to enhance the efficiency [4, 5, 6, 7]. One important instance among these tasks is the discrimination of unitary operations. Although two nonorthogonal quantum states cannot be discriminated with certainty whenever only finitely many number of copies are available [8], a perfect discrimination between two different unitary can always be achieved by taking a suitable entangled state as input and then applying only a finite number of runs of the unknown unitary operation [5, 6 ]. It is widely believed that this remarkable effect is essentially due to the use of quantum entanglement. As entanglement is a kind of nonlocal correlation existing between different quantum systems, creation of entanglement needs to perform joint quantum operations on two or more systems. These joint operations are generally difficult and expensive. Consequently, it is of great importance to consume as small amount of entanglement as possible in accomplishing a given task. This motivates us to ask: "What kind of tasks can be achieved without entanglement?"

Some pioneering works have been devoted to a good understanding of the exact role of quantum entanglement in the context of quantum computing. It has been shown that for certain problems, including DeutschJoza's problem [9], Simon's problem [10], and quantum search problem [11], quantum computing devices may still have advantages over than any known classical computing devices even without the presence of entanglement $[12,13,14,15]$. It was also argued that it may be the interference and the orthogonality but not the entanglement which are responsible for the power of quantum computing [13].

In this letter we contribute a new instance of this kind of problems in the context of quantum information by reporting a somewhat counterintuitive result: Entangle- ment is not necessary for perfect discrimination between unitary operations. We achieve this goal by explicitly constructing a simple scheme where no entanglement is needed to discriminate any two given unitary operations with certainty.

The basic idea behind our scheme can be best understood in the following scenario. Suppose we are given an unknown quantum circuit which is secretely chosen from two alternatives: $U$ or $V$. Here both $U$ and $V$ are unitary operations acting on a $d$-dimensional Hilbert space (qudit). To determine which case it really is, we first apply this circuit to a qudit initially prepared in some state $|\psi\rangle$. This action will transform the state of the system into $U|\psi\rangle$ or $V|\psi\rangle$, depending on the unknown circuit is $U$ or $V$. If there exists a suitable $|\psi\rangle$ such that the above resulting states are orthogonal, then a perfect discrimination is achieved. If such a state does not exist, we apply a suitable unitary operation, say $X_{1}$, on the above qudit and apply the unknown circuit once more. After these two runs the state of the qudit becomes $U X_{1} U|\psi\rangle$ or $V X_{1} V|\psi\rangle$. Similarly, if there exists a suitable input state $|\psi\rangle$ and unitary operation $X_{1}$ such that the resulting states are orthogonal, then a perfect discrimination is achieved again. Otherwise repeat this procedure. After $N$ runs, the final state is $\left|\psi_{U}\right\rangle=U X_{N-1} U \cdots X_{1} U|\psi\rangle$ or $\left|\psi_{V}\right\rangle=V X_{N-1} V \cdots X_{1} V|\psi\rangle$. Interestingly, there always exist a finite $N$, a sequence of unitary operations $X_{1}, \cdots, X_{N-1}$, and a suitable input state $|\psi\rangle$ such that the final output states $\left|\psi_{U}\right\rangle$ and $\left|\psi_{V}\right\rangle$ are orthogonal. See FIG. 1 for a more intuitive demonstration of this procedure.

A delicate analysis shows that the number of the runs needed in the above protocol is equal to that in the original protocol [5, 6] and is optimal in any scheme that can perfectly discriminate $U$ and $V$. It is clear in the above scheme entanglement is not used. All we need is the ability to perform unitary operations and projective measurements on a single qudit, which can be implemented efficiently and economically in experiment.

Let us begin with some preliminaries that are useful in presenting our main results. We will denote the $d$ - 


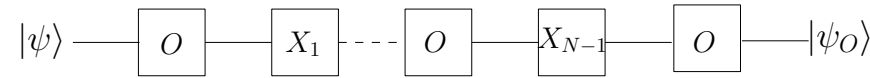

FIG. 1: A sequential scheme for discriminating unitary operations $U$ and $V$ without entanglement or joint quantum operations. Here $O$ represents the unknown circuit, $N$ is the number of the runs of applying $O, X_{1}, \cdots, X_{N-1}$ are the auxiliary unitary operations, and $|\psi\rangle$ is the input state. The output states $\left|\psi_{U}\right\rangle$ and $\left|\psi_{V}\right\rangle$ are orthogonal.

dimensional Hilbert space by $\mathcal{H}_{d}$. The notion $\mathcal{U}(d)$ represents the set of unitary operations acting on $\mathcal{H}_{d}$. When the dimension is clear from the context, we omit $d$ for simplicity. For a unitary operation $U$, we denote by $\Theta(U)$ the length of the smallest arc containing all the eigenvalues of $U$ on the unit circle. It is obvious that $\Theta(U)=\Theta\left(U^{\dagger}\right)$ and $\Theta(U)=\Theta\left(X U X^{\dagger}\right)$ for any $X \in \mathcal{U}$. We say unitary operations $U$ and $V$ are different if $U$ is not of the form $e^{i \theta} V$ for any real $\theta$.

Applying the notations introduced above, we can restate the main ideas in Refs. [5, 6] as follows. Two unitary operations $U$ and $V$ are perfectly distinguishable if and only if $\Theta\left(U^{\dagger} V\right) \geq \pi$. The perfect distinguishablity between $U$ and $V$ in the multiple-run scenario means there always exists a finite $N$ such that $\Theta\left(\left(U^{\dagger} V\right)^{\otimes N}\right) \geq \pi$, which is essentially due to the inequality $\Theta\left(W^{\otimes k}\right) \geq \min \{k \Theta(W), \pi\}$ for any unitary $W$ and $k \geq 1$. The minimal $N$ such that $\Theta\left(\left(U^{\dagger} V\right)^{\otimes N}\right) \geq \pi$ is given by $\left\lceil\frac{\pi}{\Theta\left(U^{\dagger} V\right)}\right\rceil$. Here $\lceil x\rceil$ denotes the smallest integer that is not less than $x$. The protocol that discriminates $U$ and $V$ with certainty consists of three steps: (1) Prepare an $N$-qudit input state $|\psi\rangle$; (2) Apply the unknown circuit $N$ times on $|\psi\rangle$ (each qudit one time); (3) Perform a projective measurement on the output states. Intuitively, this kind of protocol is called parallel scheme. We should point out that the input state $|\psi\rangle$ such that $U^{\otimes N}|\psi\rangle$ and $V^{\otimes N}|\psi\rangle$ are orthogonal should be an $N$ qudit entangled state. How to generate such an entangled state is a formidable task up to now even for moderately large $N$. Consequently, this kind of scheme can be implemented neither efficiently nor economically in practice.

Let us consider a different scheme. We perform the unknown circuit on the input state step by step. In contrast to the parallel scheme, this scheme is intuitively named sequential scheme. To enable the sequential scheme as powerful as possible, we insert a suitable unitary operation between each two runs of the unknown circuit. This action can adapt the output state of the previous run to be the best input state for the next run. Surprisingly, sequential scheme always leads to a perfect discrimination between any two unitary operations.

Theorem 1. Let $U$ and $V$ be two different unitary operations, and let $N=\left\lceil\frac{\pi}{\Theta\left(U^{\dagger} V\right)}\right\rceil$. Then there exist
$X_{1}, \cdots, X_{N-1} \in \mathcal{U}$ and $|\psi\rangle \in \mathcal{H}$ such that

$$
U X_{N-1} \cdots X_{1} U|\psi\rangle \perp V X_{N-1} \cdots X_{1} V|\psi\rangle .
$$

Proof. For simplicity, we consider first the case where $V$ is the identity, and then reduce the general case to this special one. We shall show the following claim: For any nontrivial $U \in \mathcal{U}(d)$ and $N=\left\lceil\frac{\pi}{\Theta(U)}\right\rceil$, there exists $X \in \mathcal{U}(d)$ such that $\Theta\left(X^{\dagger} U X U^{N-1}\right) \geq \pi$. In other words, there exists a state $|\psi\rangle \in \mathcal{H}_{d}$ such that $X|\psi\rangle$ and $U X U^{N-1}|\psi\rangle$ are orthogonal, and thus $U$ and $I$ are perfectly distinguishable by $N$ uses.

Let us consider first the case when $d=2$. By the spectral decomposition theorem, we may assume that $U$ is of the form $\operatorname{diag}\left(e^{i \theta}, 1\right)$, where $\theta=\Theta(U) \in(0, \pi]$. If $\theta=\pi$, then letting $N=1$ and $X=I_{2}$, we can directly verify the validity of the result. Otherwise, let

$$
X=\left(\begin{array}{cc}
\cos \alpha & -\sin \alpha \\
\sin \alpha & \cos \alpha
\end{array}\right)
$$

be a real rotation, where $0 \leq \alpha \leq \frac{\pi}{2}$. First we seek $\alpha$ such that $\operatorname{tr}\left(X^{\dagger} U X U^{N-1}\right)=0$, which is equivalent to

$$
\cos ^{2} \alpha e^{i N \theta}+\sin ^{2} \alpha e^{i(N-1) \theta}+\sin ^{2} \alpha e^{i \theta}+\cos ^{2} \alpha=0 .
$$

Noticing $(N-1) \theta<\pi \leq N \theta$, we can fulfil the above equation by taking

$$
\alpha=\tan ^{-1} \sqrt{-\frac{\cos (N \theta / 2)}{\cos ((N-2) \theta / 2)}} .
$$

Second, for the above $\alpha$, let $X^{\dagger} U X U^{N-1}=$ $e^{i \beta}\left|\psi_{1}\right\rangle\left\langle\psi_{1}\left|-e^{i \beta}\right| \psi_{2}\right\rangle\left\langle\psi_{2}\right|$ be the spectral decomposition. Choose $|\psi\rangle=\left(\left|\psi_{1}\right\rangle+\left|\psi_{2}\right\rangle\right) / \sqrt{2}$. It is easy to verify that $\left\langle\psi\left|X^{\dagger} U X U^{N-1}\right| \psi\right\rangle=0$.

Now for the general case $d>2$. We can assume without loss of generality that $U$ is of the form $\operatorname{diag}\left(e^{i \theta_{1}}, e^{i \theta_{2}}, \cdots, e^{i \theta_{d}}\right)$, where $0 \leq \theta_{k} \leq \Theta(U)<\pi$. In addition, we assume $\theta_{1}=\Theta(U)$ and $\theta_{2}=0$. Then applying the result in the case of $d=2$, we confirm the existence of $X_{11} \in \mathcal{U}(2)$ and $\left|\psi^{\prime}\right\rangle \in \mathcal{H}_{2}$ such that $\left\langle\psi^{\prime}\left|X_{11}^{\dagger} U_{11} X_{11} U_{11}^{N-1}\right| \psi^{\prime}\right\rangle=0$, where $U_{11}=\operatorname{diag}\left(e^{i \theta_{1}}, 1\right)$. The proof of the claim is completed by setting $X=$ $X_{11} \oplus I_{d-2}$ and $|\psi\rangle=\left|\psi^{\prime}\right\rangle \oplus 0_{d-2}$.

Let us continue the proof for the general $V$. Setting $U$ and $N$ as $U^{\dagger} V$ and $\left\lceil\frac{\pi}{\Theta\left(U^{\dagger} V\right)}\right\rceil$, respectively and applying the above claim, we have the existence of $X \in \mathcal{U}$ and $\left|\psi^{\prime}\right\rangle \in \mathcal{H}$ such that $X\left|\psi^{\prime}\right\rangle$ and $U^{\dagger} V X\left(U^{\dagger} V\right)^{N-1}\left|\psi^{\prime}\right\rangle$ are orthogonal. The proof of the theorem is completed by letting $X_{1}=X_{2}=\cdots=X_{N-2}=U^{\dagger}, X_{N-1}=X U^{\dagger}$, and $|\psi\rangle=\left|\psi^{\prime}\right\rangle$.

The above proof also presents an explicit protocol for discriminating any two unitary operations without entanglement or joint operations. It is clear that only two different auxiliary unitary operations, say, $U^{\dagger}$ and 
$X$, are required. This makes the above scheme actually feasible in experiment. It is also worth noting that the input states leading to perfect discrimination for different unitary operations are in general not the same. Interestingly, when only $2 \times 2$ unitary operations are under consideration, any maximally entangled state of the form $|\Phi\rangle=(|00\rangle+|11\rangle) / \sqrt{2}$ is a universal input. This is mainly due to the simple fact that two 1-qubit unitary operations $U$ and $V$ are perfectly distinguishable if and only if $\operatorname{tr}\left(U^{\dagger} V\right)=0$, which is also equivalent to $(I \otimes U)|\Phi\rangle \perp(I \otimes V)|\Phi\rangle[5]$. Of course, any such input state independent scheme needs to consume a maximally entangled state.

Combining the parallel scheme with the sequential scheme, we can design many different mixed schemes for discriminating unitary operations $U$ and $V$. For simplicity, let us assume $V=I_{d}$. Let $1<m<N$, and let $k_{1}, \cdots, k_{m}$ be an $m$-partition of $N$, i.e., $\sum_{i=1}^{m} k_{i}=N$, $k_{i} \geq 1$. It is clear that discriminating $U^{k_{1}} \otimes \cdots \otimes U^{k_{m}}$ and $I_{d^{m}}$ with certainty is sufficient for discriminating $U$ and $I_{d}$. A simple mixed scheme is to prepare an $m$-qudit system and then for each $1 \leq i \leq m$ apply $k_{i}$ times of the unknown circuit to the $i^{t h}$ qudit sequentially. FIG. 2 is a mixed scheme with $N=6, m=2$, and $k_{1}=k_{2}=3$.

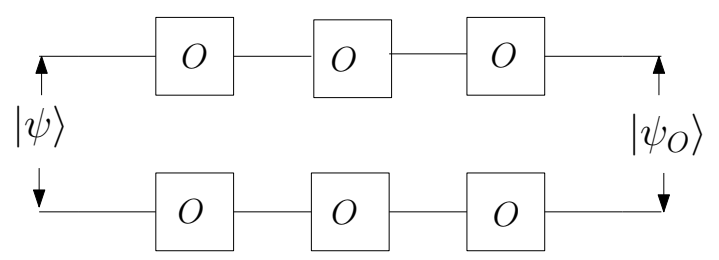

FIG. 2: A mixed scheme for discriminating $U$ and $I$. Here $O$ represents the unknown circuit, $|\psi\rangle$ is a two-qudit input state, and the output state $\left|\psi_{O}\right\rangle=\left(O^{3} \otimes O^{3}\right)|\psi\rangle$.

The validity of the scheme is essentially due to the following inequality

$$
\Theta\left(U^{k_{1}} \otimes \cdots \otimes U^{k_{m}}\right) \geq \min \left\{\left(\sum_{i=1}^{m} k_{i}\right) \Theta(U), \pi\right\} \geq \pi,
$$

which can be directly verified by the definition of function $\Theta$. Any different $m$-partition of $N$ will yield different mixed scheme. We define the length of the mixed scheme related to the partition $\left\{k_{i}\right\}$ as $\max _{1 \leq i \leq m} k_{i}$. In practice we hope the length of the scheme is as small as possible. It is not difficult to see that the minimal length can be achieved when the $m$-partition of $N$ is as uniform as possible. The minimal length is given by $n_{\min }=\left\lceil\frac{N}{m}\right\rceil$. Let $N=\left(n_{\min }-1\right) m+r$ for some $1 \leq r \leq m$. Then a corresponding partition is $k_{1}=\cdots=k_{r}=n_{\text {min }}$, $k_{r+1}=\cdots=k_{m}=n_{\text {min }}-1$.

Let us give some remarks about different schemes for discrimination. The most advantage of the sequential scheme is that no entanglement or joint quantum operations is needed. However, any such kind of scheme needs to perform sequentially at least $N$ times of the unknown circuit. Instead, in the parallel scheme one needs to prepare an $N$-partite entangled state as probe state (Here we notice that it is possible to discriminate two $N$-partite orthogonal states $U^{\otimes N}|\psi\rangle$ and $V^{\otimes N}|\psi\rangle$ by using local operations on each single qudit and classical communications between different qudits only [16], so the measurement device does not require joint quantum operations). When there are at least $N$ copies of the unknown circuit and suitable entanglement, we can complete the discrimination within a single step by applying $N$ copies of the unknown circuit to the input state simultaneously. For the case when only $1<m<N$ copies of the unknown circuit are available, the discrimination task can be finished in $\left\lceil\frac{N}{m}\right\rceil$ steps. This reveals an interesting tradeoff between the spatial resources (entanglement or circuits) and the temporal resources (running steps or discriminating time). One should choose the most economic scheme in order to save the resources which are crucial in practice.

We notice that in the above schemes both the input state $|\psi\rangle$ and the measurement device for discriminating the final output states $\left|\psi_{U}\right\rangle$ and $\left|\psi_{V}\right\rangle$ are determined by $U$ and $V$. When no a priori classical information about the unknown circuit is available, the task is reduced to quantum operation estimation and it is never possible to achieve a perfect identification when only finitely many runs (copies) of the unknown circuit are allowed(available) [5]. We would also like to point out that all the above schemes require the ability of performing local operations (unitary operations or projective measurements) on a single qudit in order to perfectly discriminate the output states. This fact is a little surprising as it seems that the parallel scheme does not need any auxiliary unitary operations.

For parallel scheme it has been shown that $N=$ $\left\lceil\frac{\pi}{\Theta\left(U^{\dagger} V\right)}\right\rceil$ is the optimal number of the runs to achieve a perfect discrimination between $U$ and $V[5]$. In what follows we shall prove that this number is also optimal for perfect discrimination between $U$ and $V$ by using any sequential scheme. To present this result, we first introduce a key lemma.

Lemma 1. Let $U$ and $V$ be two unitary operations such that $\Theta(U)+\Theta(V)<\pi$. Then $\Theta(U V) \leq \Theta(U)+\Theta(V)$.

It is interesting that Lemma 1 can be directly derived from Lemma 3 in Ref. [4]. So we omit the proof here.

Theorem 2. Let $U$ and $V$ be two different unitary operations, and $k<\left\lceil\frac{\pi}{\Theta\left(U^{\dagger} V\right)}\right\rceil$. Then for any unitary operations $X_{1}, \cdots, X_{k-1} \in \mathcal{U}$ and $|\psi\rangle \in \mathcal{H}$,

$$
U X_{k-1} \cdots X_{1} U|\psi\rangle \not \perp V X_{k-1} \cdots X_{1} V|\psi\rangle .
$$

Proof. Without any loss of generality we may assume that $V=I$ as it is clear that discriminating $U$ and $V$ is equivalent to discriminating $U^{\dagger} V$ and the identity $I$. 
To prove Theorem 2, it is sufficient to show that if $k<N=\left\lceil\frac{\pi}{\Theta(U)}\right\rceil$ then for any $X_{1}, \cdots, X_{k-1} \in \mathcal{U}$, we have

$$
\Theta\left(\left(X_{k-1} \cdots X_{1}\right)^{\dagger}\left(U X_{k-1} \cdots X_{1} U\right)\right)<\pi .
$$

Applying Lemma 1 $(k-1)$ times we have the following

$$
\Theta\left(\left(X_{k-1} \cdots X_{1}\right)^{\dagger}\left(U X_{k-1} \cdots X_{1} U\right)\right) \leq k \Theta(U),
$$

where we have used the fact that $\Theta\left(X^{\dagger} U X\right)=\Theta(U)$ for any unitary $X$. Noticing that $k \leq N-1$ and $(N-1) \Theta(U)<\pi$, we have the validity of Eq. (1).

Employing the similar techniques, we can easily show that $N$ is also the minimal number of the runs of unknown circuit in any scheme (sequential scheme, parallel scheme, or any mixed scheme) that perfectly discriminates $U$ and $V$.

It is straitforward to show that any $n>2$ different unitary operations can be perfectly distinguishable without entanglement or joint quantum operations. Let $U_{1}, \cdots, U_{n}$ be $n$ possible candidates, and let $N_{i j}=$ $\left\lceil\frac{\pi}{\Theta\left(U_{i}^{\dagger} U_{j}\right)}\right\rceil$, where $1 \leq i<j \leq n$. By assuming the circuit is in $\left\{U_{1}, U_{2}\right\}$ and then applying the sequential scheme, we can reduce at least one candidate and only need to consider the left $n-1$ ones. Repeating this process at most $n-1$ times, we complete the discrimination without entanglement or joint operations. The total number of the runs $N$ satisfies $N_{\max } \leq N \leq(n-1) N_{\max }$, where $N_{\max }=\max \left\{N_{i, j}\right\}$. However, in some special cases the procedure described above is far from the optimal one. An interesting example is as follows.

Let $\{|0\rangle, \cdots,|d-1\rangle\}$ be an orthonormal basis for $\mathcal{H}_{d}$. Consider the set of $d^{2}$ generalized Pauli matrices $\left\{\sigma_{m n}\right.$ : $0 \leq m, n \leq d-1\}$, where $\sigma_{m n}=\sum_{k=0}^{d-1} \omega^{n k}|k+m\rangle\langle k|$ and $\omega=e^{\frac{2 \pi i}{d}}$. One can readily verify that any $d \times d$ maximally entangled state can be used to perfectly discriminate this set of unitary operations. How many runs are needed if the use of entanglement or joint operations is forbidden? It is obvious that a single run is not sufficient as there cannot be $d^{2}$ orthogonal states in a $d$-dimensional state space. Applying the procedure described above a $d^{2}-1$ upper bound can be obtained. We can do much better by employing a more efficient protocol. Let $\{|\bar{l}\rangle: 0 \leq l \leq d-1\}$ be another orthonormal basis such that $|\bar{l}\rangle=\frac{1}{\sqrt{d}} \sum_{k=0}^{d-1} \omega^{k l}|k\rangle$. Then it is easy to see that $\left(\sigma_{m n} \otimes \sigma_{m n}\right)|0 \overline{0}\rangle=\omega^{-m n}|m \bar{n}\rangle$. Intuitively, by measuring the first qudit we obtain the index $m$, while by measuring the second qudit we know the index $n$. Therefore two runs are necessary and sufficient to discriminate $d^{2}$ Pauli matrices without entanglement or joint operations whenever how large $d$ is. This example also demonstrates that entanglement may reduce the number of the runs when discriminating $n>2$ unitary operations.
In conclusion, we present a sequential scheme using only unitary operations and projective measurements to perfectly discriminate unitary operations. No entanglement or joint quantum operations is required. This implies that entanglement is not essential in achieving the perfect discrimination between unitary operations, and in some sense, confirms the importance of interference and orthogonality, as suggested in Ref. [13]. We also propose various mixed schemes for discrimination and show the optimality of these schemes. Notably, there exists an interesting tradeoff between the spatial resources and the temporal resources. These results would be helpful when we try to achieve a perfect discrimination with the lowest cost.

We thank Tal Mor, Zhengfeng Ji, and Guoming Wang for inspiring suggestions and helpful comments. The acknowledgement is also given to the other colleagues in the Quantum Computation and Quantum Information Research Group for enjoyable conversations. This work was partly supported by the Natural Science Foundation of China (Grant Nos. 60621062, 60503001, and 60433050), the Tsinghua Basic Research Foundation (Grant Nos. 052220204 and 052420003), and the Hi-Tech Research and Development Program of China (Grant No. 2006AA01Z102).

* Electronic address: dry@tsinghua.edu.cn

† Electronic address: feng-y@tsinghua.edu.cn

¥ Electronic address: yingmsh@tsinghua.edu.cn

[1] M. A. Nielsen and I. L. Chuang, Quantum Computation and Quantum Information, Cambridge University Press, Cambridge, 2000.

[2] C. H. Bennett and S. J. Wiesner, Phys. Rev. Lett. 69, 2881 (1992).

[3] C. H. Bennett, G. Brassard, C. Crepeau, R. Jozsa, A. Peres, and W. K. Wootters, Phys. Rev. Lett. 70, 1895 (1993).

[4] A. M. Childs, J. Preskill, and J. Renes, J. Mod. Opt. 47, 155 (2000).

[5] A. Acín, Phys. Rev. Lett. 87, 177901 (2001).

[6] G. M. D'Ariano, P. LoPresti, and M. G. A. Paris, Phys. Rev. Lett. 87, 270404 (2001).

[7] Z. F. Ji, Y. Feng, R. Y. Duan, and M. S. Ying, Phys. Rev. Lett. 96, 200401 (2006).

[8] A. Chefles, Physical Review A 64, 062305 (2001).

[9] D. Deutsch and R. Jozsa, Proc. R. Soc. London, Ser. A 439, 553 (1992)

[10] D. R. Simon, SIAM journal on computing 26, 1474 (1997).

[11] L K. Grover, Phys. Rev. Lett. 79, 325 (1997); Phys. Rev. Lett. 80, 4329 (1998).

[12] S. Lloyd, Phys. Rev. A 61, 010301(R) (1999).

[13] D. A. Meyer, Phys. Rev. Lett. 85, 2014 (2000).

[14] E. Biham, G. Brassard, D. Kenigsberg, and T. Mor, Theor. Comput. Sci. 320, 15 (2004).

[15] D. Kenigsberg, T. Mor, and G. Ratsaby, "Quantum Advantage without Entanglement," (2005). Report No: 
quant-ph/0511272

[16] J. Walgate, A. J. Short, L. Hardy, and V. Vedral, Phys.
Rev. Lett. 85, 4972 (2000). 